\title{
Grid-scale evaluation of five reference evapotranspiration methods based on the climate forecast system reanalysis data
}

\author{
Tekalegn Ayele Woldesenbet ${ }^{1}$ and Nadir Elagib ${ }^{2}$ \\ ${ }^{1}$ Ethiopian Institute of Water Resources \\ ${ }^{2}$ Independent Researcher
}

November 16, 2020

\begin{abstract}
Climate Forecast System Reanalysis data offer a promising database for overcoming the limitations in availability and reliability of climatological data and, hence, for understanding the hydrological processes. Using these data on grid-by-grid, seasonal and yearly scales, the present study attempts to advance the spatiotemporal evaluation of two radiation-based (Priestley-Taylor and Makkink) and three temperature-based (Hargreaves-Samani, Thornthwaite and Blaney-Criddle), against estimates of grass reference evapotranspiration (ETo) by FAO Penman-Monteith method (FAO-PM). The analysis was performed for the period 1979-2013, considering the second largest (79,000 km2) river system in Ethiopia, i.e. Omo-Gibe basin, which accommodates national parks and vast hydropower, cultivation and afforestation developments and discharges its flow to Lake Turkan in Kenya. To comprehensively explain the pattern of PET, the influences of temperatures, rainfall, wind speed, radiation, relative humidity and elevation on PET were also examined. The results emphasize the outperformance of Hargreaves-Samani method. In overall, both the annual and seasonal FAO-PM estimates are captured by this method for most of the grid locations. Annual trends in ETo in the upper region increased but rainfall trends decreased. These trends might negatively impact the rain-fed food production by reducing soil moisture availability in the river basin. Comparatively, trends in rainfall in the middle and lower regions increased with a higher magnitude while ETo increased with a smaller magnitude compared. The above-mentioned trends in ETo are attributable to rising temperature and decreasing relative humidity, wind speed, and solar radiation, respectively. If these trends would continue, we would expect increase in soil moisture for sugarcane plantation in the middle and lower region and attenuation of water loss from reservoirs in the river basin. This study improves the understanding of the best potential evapotranspiration methods in similar data-scarce river basins in Ethiopia or other transboundary rivers in the region or worldwide.
\end{abstract}

\section{Hosted file}

Main document.pdf available at https://authorea.com/users/376167/articles/493225-grid-scaleevaluation-of-five-reference-evapotranspiration-methods-based-on-the-climate-forecastsystem-reanalysis-data

\section{Hosted file}

List of Tables.pdf available at https://authorea.com/users/376167/articles/493225-grid-scaleevaluation-of-five-reference-evapotranspiration-methods-based-on-the-climate-forecastsystem-reanalysis-data 

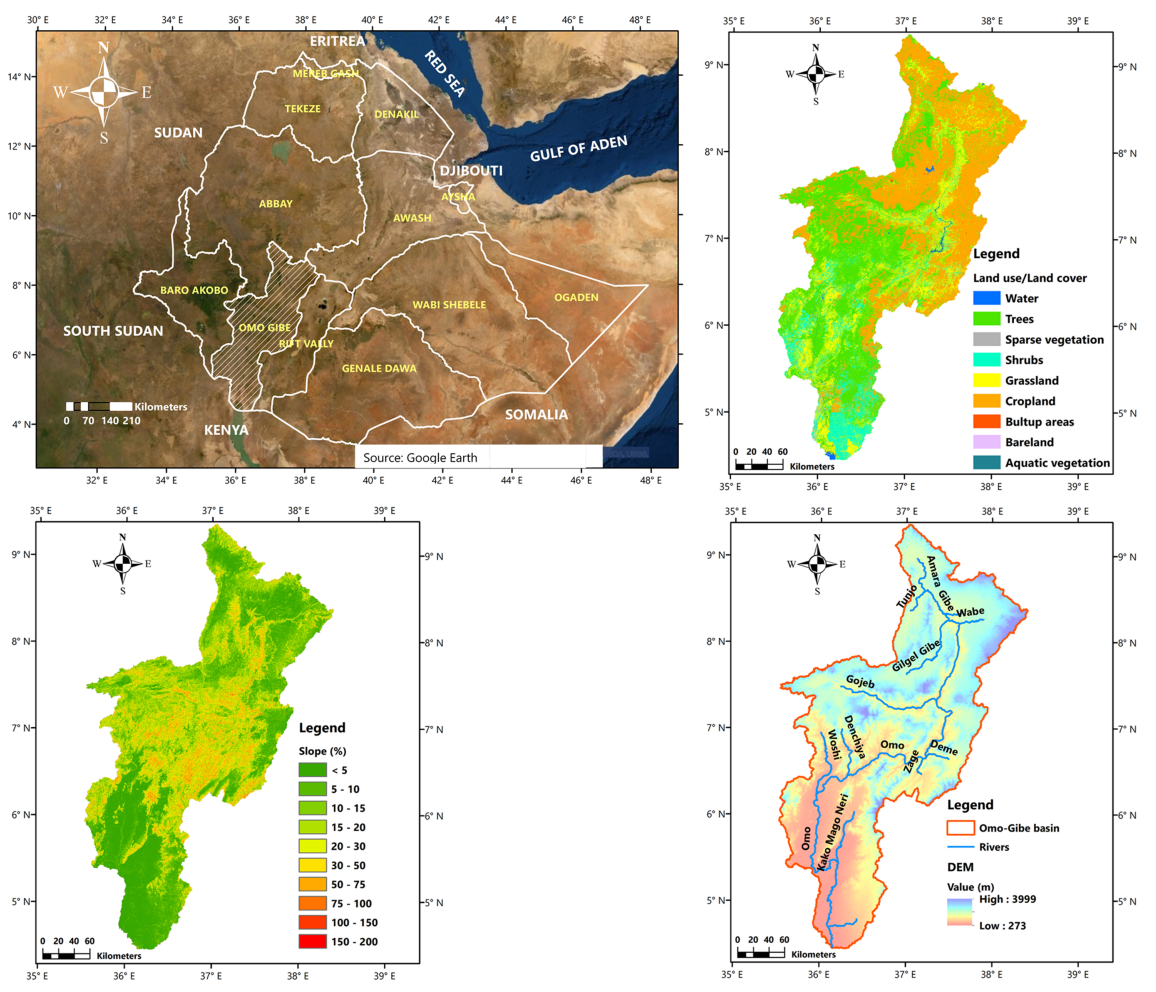


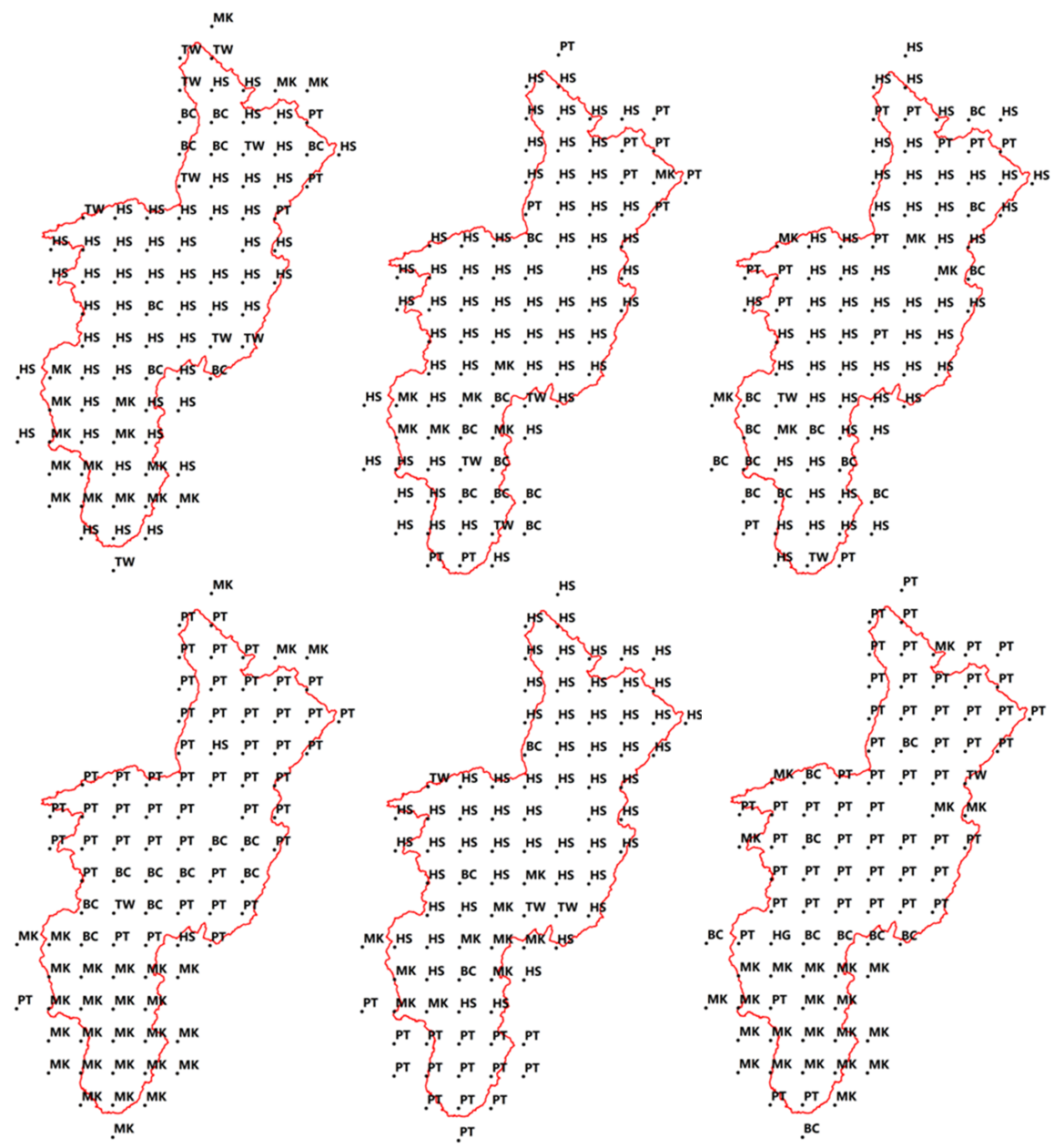

a) Kendall correlation

b) Percent Bias Error

C) Root Mean Square Error 


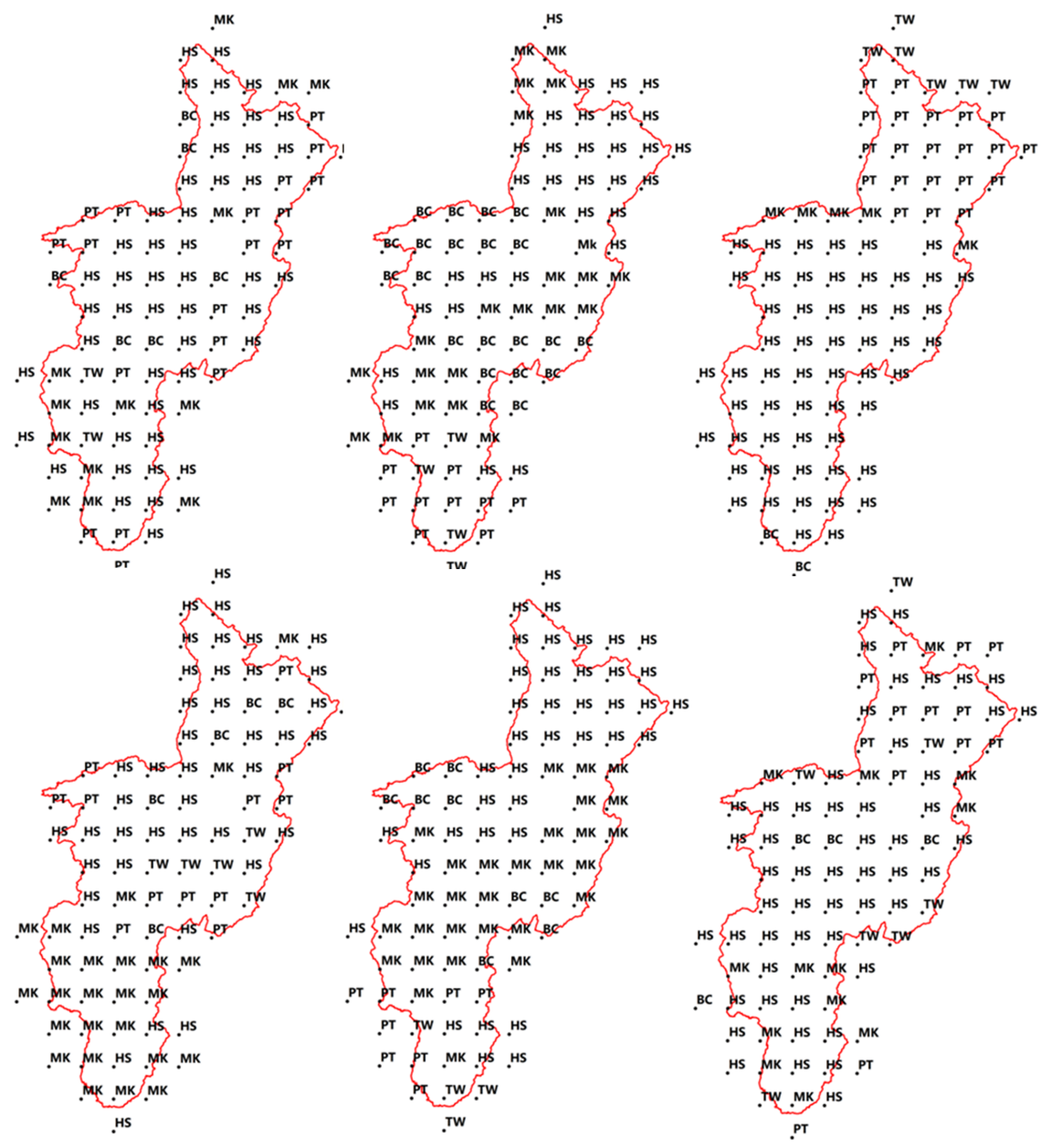

a) Kendall correlation

b) Percent Bias Error

C) Root Mean Square Error 

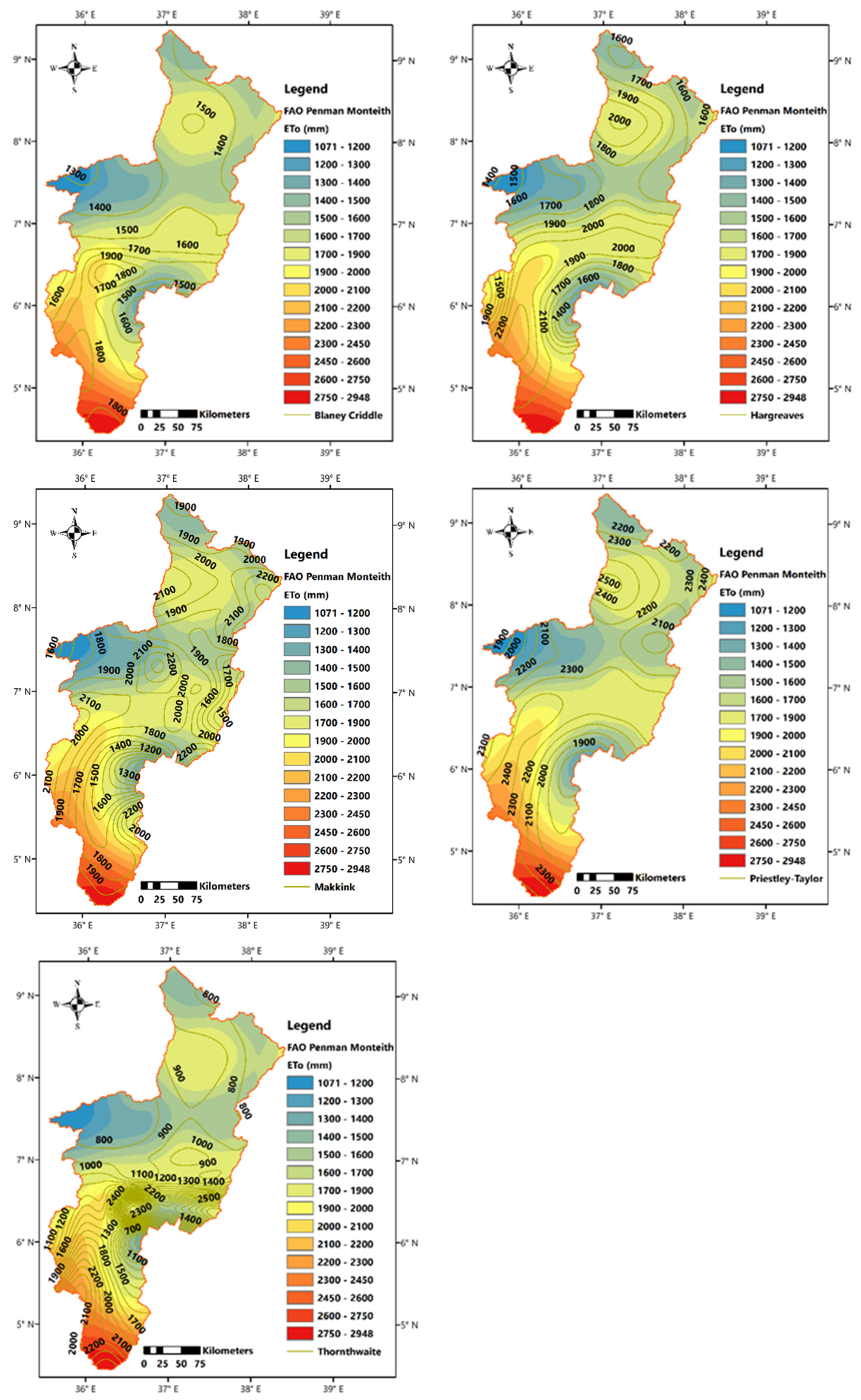
BC

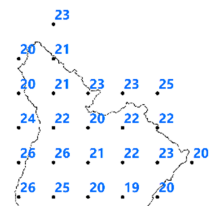

$18 \div .23 \quad .23 \cdot .22 \quad .22 \quad 20 \quad .95$

$\begin{array}{lllllll}513 & 17 & 20 & .^{20} & .^{19} & .19 & 48\end{array}$

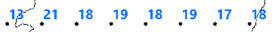

$\begin{array}{llllll}316 & .^{18} & .20 & .20 & .^{19} & .18\end{array}$

$\begin{array}{llllll}18 & .^{18} & .^{22} & .^{25} & .4 & .26\end{array}$

$.14 \quad \begin{array}{lllll}17 & .17 & .17 & 20 & 90\end{array}$

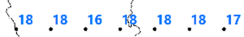

$\begin{array}{lllll}.15 & 18 & .17 & .15 & .16\end{array}$

$.^{16} .16 .16 .16 .{ }^{16}$

$.15 \quad .7 \quad .^{16} \quad .6 \quad .16$

. the 16
TW

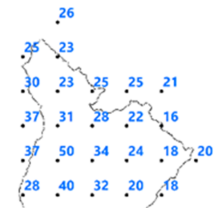

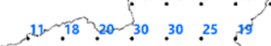

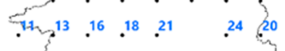

$\begin{array}{llllllll}.14 & 30 & .19 & .21 & .27 & .33 & .29 & .^{25}\end{array}$

$\begin{array}{llllll}31 & 39 & 42 & .42 & .38 & 33\end{array}$

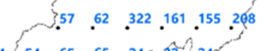

$.34 \lcm{54} \quad 65 \quad 65 \quad .24 \times 22 ? .24$

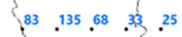

$.65 \quad 2_{13} .157 .88 \quad .56$

$. .^{124} \cdot 7^{46} \cdot .^{102} \cdot 7 x \cdot{ }^{67}$

.95 . 129.126 .974

.136 .164 .127

MK

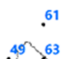

$4^{49} \sqrt{63}$

$.52 \quad .56 \backslash 59 \quad .59 \quad .65$

53) $47 \quad 40 \quad 46 \quad 50$

$\begin{array}{llllll}.56 & .58 & .45 & .46 & .49 & .43\end{array}$

$\begin{array}{llllll}55 & 53 & .45 & .42 & 43\end{array}$

$\begin{array}{rlllll}28-32 & 39 & 51 & 51 & .44 & 41\end{array}$

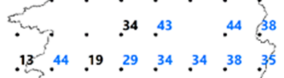

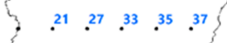

$\begin{array}{llllll}3.11 & .20 & .32 & .34 & 32 & .24\end{array}$

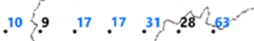

$8 \quad .11 \quad .12 \quad .293 .20$

2. 6.10

- $\cdot .7^{7}$

$.^{-4} \cdot 8 \cdot .^{5}$

$-2$.
HS

.73

\section{.657}

55 $54 \quad 59 \quad 65 \quad 67$

$\begin{array}{llllll}.56 & 53 & .57 & .65 & .73 \\ .71\end{array}$

$\begin{array}{llllll}63 & 55 & 60 & 56 & 64\end{array}$

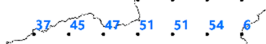

$\begin{array}{lllllll}25 & 27 & 36 & 39 & 39 & .51 & 50\end{array}$

$\begin{array}{rrrrrrr}49 & .26 & 33 & .40 & .40 & .44 & 42\end{array}$

$\left\{\begin{array}{lllll}23 & .29 & .39 & .43 & .43\end{array}\right.$

$\left\{\begin{array}{llllll}.35 & .39 & 43 & .39\end{array}\right.$

\{. $\quad .26 \quad .64 .34$

.20 .19

- $282^{24} \cdot$

- . 28.

.29 38.45

.42

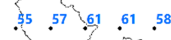

PT

.25

to $\quad .17 \quad .17 \quad .17$.

$.12 .7 \div$

.8 .9$.

$.15 \quad .^{15}$

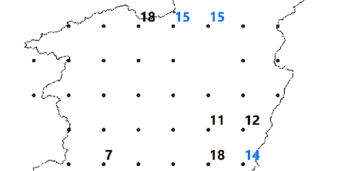

${ }^{7} \cdot .^{18} .^{14}$

$\left\{\begin{array}{lll}\cdot & \cdot & \cdot \\ \cdot & \cdot & \cdot\end{array}\right.$

i. $\cdot$

$\cdot\{\cdot$

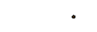

FAO-PM

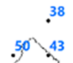

$.40 \quad .37 \quad 36 \quad 36$. .24.22.20? .14 .15 .21 .3 $\begin{array}{llll}17 & 23 & 26\end{array}$

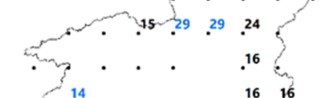

$\cdot 5^{14} \cdot \cdot . .^{16} . .^{16}$

\{. . . . .18

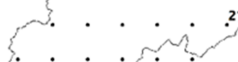

?. . .

ind $\cdot$.

$\cdot \cdot \cdot \cdot \cdot$ 


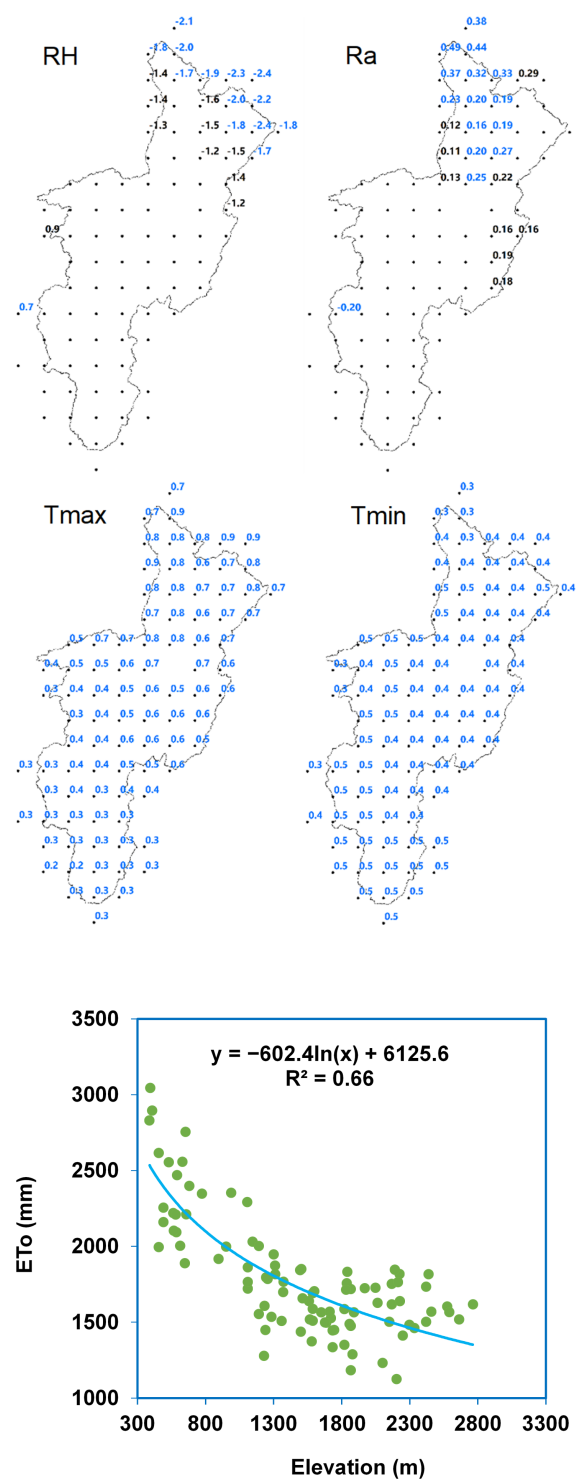

(a)
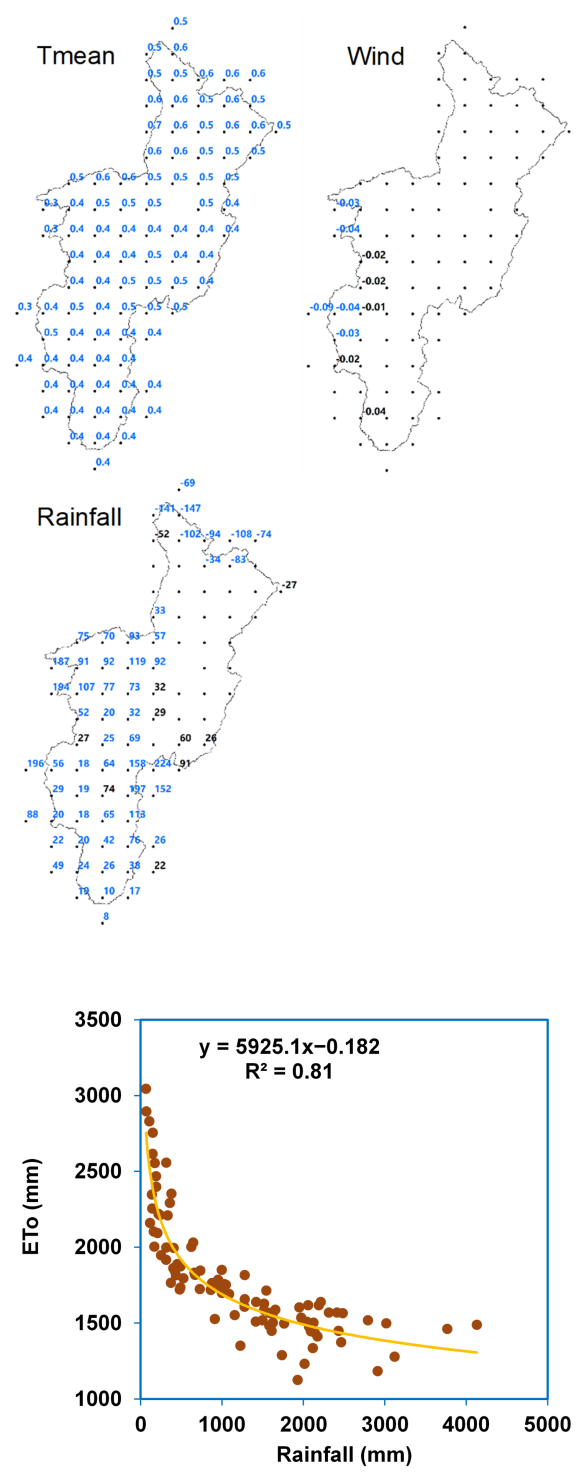

(b) 